\title{
A Prognostic Model Based on TMB-Related Lncrnas Predicts Outcome of Liver Cancer Patients
}

\section{Yao Wang}

Department of General Surgery, First Affiliated Hospital of Anhui Medical University

Chaisheng Tan

Department of Oncology, First Affiliated Hospital of Anhui Medical University

Yeben Qian ( $\nabla$ qianyeben123@hotmail.com )

Department of General Surgery, First Affiliated Hospital of Anhui Medical University

\section{Research Article}

Keywords: TMB, IncRNA, Hepatic carcinoma

Posted Date: January 25th, 2021

DOI: https://doi.org/10.21203/rs.3.rs-148944/v1

License: (c) (1) This work is licensed under a Creative Commons Attribution 4.0 International License. Read Full License 


\section{Abstract}

TMB-related Incrnas and their clinical significance in liver cancer have not been explored.Combining the expression levels of these Incrnas, somatic mutation files and clinical information in patients with liver cancer,we identified 514 Incrnas that are closely related to TMB.Based on these Incrnas, a TMB-derived IncRNA Signature (TMBLncSig) was established. TMBLncSig categorized the patients into high-risk group and low-risk group. There was a significant difference in the prognosis of the patients between the two groups, which was further verified in the independent test set.TMBLncSig is associated with TMB level in liver cancer patients and has the potential to be used as a TMB level measurement tool. In summary this st provide new thought for further study on the role of IncRNA in the difference of TMB level in liver cancer patients.

\section{Introduction}

Primary liver cancer is the second leading cause of cancer-related death worldwide and therefore a major public health challenge [1].Long-term survival and poor prognosis are associated with liver cancer, which kills more than 700,000 people worldwide each year [2].In the current clinical work, the clinical information of patients is mined to predict the disease progression and prognosis of patients, such as age, tumor stage, tumor grade, lymph node involvement.Some studies have pointed out that hepatocellular carcinoma has heterogeneity, which is reflected in the molecular level of clinical traits, as well as the occurrence, development and prognosis of hepatocellular carcinoma.

Turmor with high TMB is generally considered to be increased in neo-antigen burden, so this kind of tumor may benefit more from immunotherapy[3].Moreover, HCC patients with low TMB levels tend to have a better prognosis than those with high TMB levels[4].Long non-coding RNAs (IncRNAs) do not encode proteins and are larger than $200 \mathrm{nt}$ [5].Although IncRNAs do not have the ability to encode proteins, recent studies have shown that they are involved in a variety of biological processes[6].Lncrnas also play a important role in the development and metastasis of tumors[7].With the development of gene sequencing technology, it is gradually found that there are significant differences in the expression levels of a large number of Incrnas in different types of tumors, but the functions of most of them are still unknown[8]. With the development of research, people realize that IncrNA also plays an important role in Tumor mutational burden (TMB) .For example, IncRNA DDSR1 participates in DNA damage Response (DDR), and DDR variation will lead to TMB variation.LncRNA NORAD helps to maintain genomic stability, which also affects TMB size[9][10]. However, the relationship between these tumor mutational burdenrelated Incrnas and the clinical progress of patients has not been further explored.In this study LncRNAs related to TMB was identified based on TCGA database,and their ability to indicate TMB level and predict prognosis in patients with liver cancer was explored.

\section{Materials And Methods}

\section{Data collection}


We collected Clinical information $₫ T$ Transcriptome high throughput sequencing data and somatic mutation data of liver cancer patients form TCGA database (https://portal.gdc.cancer.gov/) [11-12]. We annotated the transcriptome high-throughput sequencing data using GRCH38[13](last-updated 2019-06) then we obtained the expression of Long non-coding RNA.Among these data, 369 patients with corresponding clinical information, somatic mutation data and IncRNA expression level were selected for further analysis.These patients were divided into the training group and the verification group, which consisted of 185,184 patients respectively $₫$ table $1 \otimes$. The clinical and pathological characteristics of the patients in TCGA data sets are summarized in table 1.

\section{Identification of TMB-associated IncRNAs}

TMB is calculated as the total number of somatic mutations (including mutations, insertion and deletion of non-synonymous points in exon coding region)/the size of target region, on the unit of mutations/Mb[14].The TMB of each patient's tumor sample was calculated, and the patients were then sorted in TMB size order.The top $25 \%$ of patients were defined as the TMB-high group, while the bottom $25 \%$ were defined as the TMB-low group.The expression profiles of Incrnas in the TMB-high and TMB-low groups were obtained by limMA method (fold change $>2$ or $<0.5$ and false discovery rate (FDR) adjusted $P<0.05)$, and these were defined as TMB-related Incrnas.

\section{Statistical analysis}

Methods of hierarchical cluster analysis are used in this study ,and we used Euclidean distances and Ward's linkage Method to complete a hierarchical Cluster analysis[15].The relationship between TMB associated IncRNAs and overall survival was calculated by univariate and multivari Cox proportional hazard regression analysis. Through the above process, TMB-associated IncRNAs related to prognosis were obtained,then a TMB-derived IncRNA Signature (TMBLncSig) was constructed.TMBLncSig score of each patient is shown in the following formula:

TMBLncSig $=0.403 * A C 128709.3+1.026 * C 10$ orf126-0.504*DIO3OS+0.557*HOXD -

AS2+0.554*LINC00668+0.593 *LINC00707+0.098 *MIR210HG-3.362*RP1-47M23.3+6.588*ZEB2-

AS1+1.158*RP11-437L7.1.

TMBLncSig is a prognostic risk score for the liver cancer patient. The IncRNAs in the above formula represent their expression levels in the patient samples. The coefficient multiplied before each IncRNA represents the contribution of this IncRNA to the prognostic risk score obtained by multivariate cox analysis of regression coefficients.

In the training group, the median score was used to divide patients into two groups (high-risk group with high TMBLncSig or low-risk group with low TMBLncSig).

Product-limit method is selected to calculate survival rate and median survival.We used log-rank test to explore whether there is a difference between the survival of the high-risk group and the low-risk group[16].P value less than 0.05 means that the difference between the two groups is 
significant[17].TMBLncSig's predictive efficacy of patient survival is evaluated by the time-dependent receiver operating characteristic (ROC) curve[18].All the above statistical analyses were completed by $\mathrm{R}$ (version 4.0.3).

\section{Results}

\section{Identification of TMB-related IncRNAs in liver cancer patients}

We calculated the Tumor Mutational burden of all Tumor samples from TCGA patients with liver cancer. The patients were sorted by TMB, and the top $25 \%$ of patients with the largest $\operatorname{TMB}(n=90)$ were treated as the high-TMB group, and the $25 \%$ with the smallest TMB $(n=88)$ were treated as the low-TMB group."Limma" package of "R" software was used to screen the differential genes between the low-TMB group and high-TMB group[19].According to the threshold set by us(|FC|ख2冈FDR囚0.05), a total of 514 Incrnas were considered to have different expression levels in the two groups.Among them, 313 IncRNAs were up-regulated in the low-TMB group, and 201 IncRNAs were up-regulated in the high-TMB group(Supplementary Table 1).

Unsupervised hierarchical clustering analysis was carried out for a total of 374 tumor samples using these 514 IncRNAs(Figure 1A)[15]. The samples were clustered into two groups using the above method.Because the TMB of the samples between the two groups is significantly different, we define the two groups as lowTMB-like group and HightMB-like group.The TMB's median value of the lowTMB-like group is significantly higher than that of the HightMB-like group (3.316 vs 1.882,P囚0.001囚 Mann-Whitney U test; Figure 1B).

\section{Development of a TMB-derived IncRNA signature for outcome prediction in the training set}

In order to explore the effect of these TMB-related IncRNAs on the prognosis of liver cancer patients, we matched the expression levels of these IncRNAs with the clinical information of patients in the training group and the verification group. In the training group, we used univariate cox proportional regression analysis and found that 33 TMB-related IncRNAs were significantly associated with the prognosis of liver cancer patients $(P<0.05$,Supplementary Table 2$)$. The multivariate cox proportional hazard regression analysis was performed on these genes and 10 Incrnas obtained. The TMB-derived IncRNA Signature (TMBLncSig) was constructed to predict the prognosis of liver cancer patients according to the multivariate Cox analysis coefficient and the expression level of these 10 TMB-related IncRNAs(Supplementary Table 3). TMBLncSig $=0.403 * A C 128709.3+1.026 *$ C10orf 126$-0.504 *$ DIO3OS+0.557*HOXD-AS2+0.554*LINC00668+0.593 *LINC00707+0.098*MIR210HG-3.362*RP147M23.3+6.588*ZEB2-AS1+1.158*RP11-437L7.1.

The TMB-derived IncRNA Signature (TMBLncSig) was used to obtain the risk score of each liver cancer patient in training set, and the patients were divided into two groups according to their median value as the dividing line(0.918).The group with TMBLncSig score above 0.918 was called high risk group, while the group with TMBLncSig score below 0.918 was called low risk group. 


\section{Kaplan-meier analysis was used to describe the difference in prognosis between the two groups.}

The survival outcomes of low risk Group is better than that of high risk Group (Figure 2A》 median OS 2.1 years versus 1.2 years, $p<0.001$,log-rank test). Time-dependent ROC curves analysis was used to evaluate TMBLncSig's predictive ability of prognosis. The AUCs for the 1,2,3,4,5year survival were $0.73,0.8,0.8,0.81,0.84$ in training group(Figure $2 B$ ). Through multivariate analyses, TMBLncSig was significantly associated with patient's OS (HR=1.162,Pख0.001).

In the training group, all patients were ranked from low to high according to TMBLncSig, and the difference of TMB between the low risk group and the high risk group was observed(Figure 2c,Figure 2D).It showed that There were significant differences in TMB between high and low risk groups.As the figure shows, the TMB of the high-risk group is higher than that of the low-risk group(median TMB 3.592 versus 2.842, $\mathrm{P}<0.001$, Mann-Whitney $\mathrm{U}$ test).Lncrna RP1-47M23.3 and DI030S were highly expressed in the TMBLncSig low-risk group and Lncrnas AC128709.3,C10orf126,HOXDAS2,LINC00668,LINC00707,MIR210HG,ZEB2-AS1,RP11-437L7.1 were highly expressed in the TMBLncSig high-risk group.

\section{Independent validation of TMBLncSig in the liver cancer data set with RNA-seq platform}

To verify whether TMBLncSig can be used for other samples that are not in the training set,We tested its predictive power in the validation set (184 patients of TCGA database). With the same riskscore cutoff as the training set, the verification set was divided into the high-risk group and the low-risk group with 105 and 79 pantients respectively. The high risk group had a worse prognosis than the low risk group (Figure $3 \mathrm{~A}$, median OS 0.83

versus 1.35 years, $P=0.008$, log-rank test). The high risk group had a lower one-year survival rate than the low risk group(71\% versus $87 \%$ ). The AUC for The 1 year survival were 0.662 in test group through time-dependent ROC curves analysis(Figure 3C). In the validation set, there were significant differences in TMB between the high and low risk groups (median 3.41 versus 2.47, P $<0.001$, Mann-Whitney U test; Figure 3G).

In all TCGA liver cancer patients, TMBLncSig still has a good predictive effect on the prognosis of patients. We still took 0.918 as the cutoff value and divided all patients in the TCGA set into the high-risk $\operatorname{group}(n=171)$ and the low-risk $(n=198)$ group. Similar to the training set validation set, the high risk TCGA group had a worse prognosis than the low risk TCGA group( median survival 1.08 versus 1.52 years, $P=\square$ 0.001 log-rank test; Figure 4B).The high risk group had a lower one-year survival rate than the low risk group(74\% versus $91 \%)$. The AUC for The 1 year survival were 0.679 in TCGA set through time-dependent ROC curves analysis(Figure 4D). In the TCGA set, there were significant differences in TMB between the high and low risk groups (median 3.5 versus 2.6, $\mathrm{P}<0.001$, Mann-Whitney $U$ test; Figure $4 \mathrm{H}$ ).

\section{The TMBLncSig predicts outcome better than TP53 mutation status}


There are significant differences in the proportions of TP53 mutations over TMBLncSig high-risk and lowrisk groups. This is similar in the training set, validation set and TCGA set (Figure 5A). In the training set, the proportion of TP53 gene mutations in the samples of patients in the high-risk group was $42 \%$, and that in the low-risk group was $26 \%$ (chi-square test $P=0.039$ ). In the validation set,the proportion of TP53 gene mutations in the samples of patients in the high-risk group was $38 \%$, and that in the low-risk group was $19 \%$ (chi-square test $P=0.007$ ). A similar phenomenon was observed in the TCGA set,In the TCGA set,the proportion of TP53 gene mutations in the samples of patients in the high-risk group was $40 \%$, and that in the low-risk group was $22 \%$ (chi-square test $P \otimes 0.001$ ). This shows that TMBLncSig is also closely related to TP53 gene mutation. Many literatures have shown that TP53 predicts poor prognosis in liver cancer patients. Therefore, we further observed the prediction of TMBLncSig and TP53 mutation on the prognosis of liver cancer patients. Through TMBLncSig and TP53 mutations, we divided TCGA set HCC patients into four groups. We refered to patients with TP53 mutations and high TMBLncSig risk value as TP53 Mutation/high risk group( $n=66)$. And by doing that, we got TP53 Mutation/low risk group $(n=43)$, TP53 Wild/high risk group $(n=98)$ and TP53 Wild/low risk group $(n=150)$. The survival curves of the four groups are shown in the Figure 5B. Patients in the TP53 Wild/low risk group had the best prognosis, while patients in the TP53 Mutation/high risk group had the worst prognosis(median OS 1.71 years versus 0.833 years, $P=0.01$, log-rank test). The ability of TP53 mutation to predict the prognosis of liver cancer patients is limited, and combination with TMBLncSig can achieve better prognosis prediction effect.

\section{Independence of the TMBLncSig from other clinical factors}

Stratification analysis was used to validate the Independence of the TMBLncSig from other Clinical factors.Patients of TCGA Set were divided into young group $(n=177)$ and old group $(n=192)$ at the median age $(a g e=60)$. Patients in each group were divided into high-risk and low-risk groups by TMBLncSig,and there were significant differences in OS between the high-risk and low-risk groups in both young group (log-rank test $P \otimes 0.001$; Figure $4 \mathrm{~A}$ ) and old group(log-rank test $\mathrm{P} \otimes 0.001$; Figure $4 \mathrm{~B}$ ). Then patients in TCGA set were divided into early-stage groups (stage I or II $\llbracket n=255$ ) and late-stage group (stage III or IV $\llbracket n$ = 90).TMBLncSig classified the early-stage and late-stage groups into high-risk group and low-risk group respectively. The OS differences between low risk groups and high risk group of early-stage and late-stage groups were shown in Figure $4 \mathrm{C}$ and Figure 4D respectively. The OS was significantly different between the two groups.We used Grade and Tumor status to perform the same operation above, and the results were shown in Figure 4E,Figure 4F,Figure $4 \mathrm{G}$ and Figure $4 \mathrm{H}$. TMBLncSig has a good ability to predict the prognosis of patients in early-Tumor status group (T1 or T2, n=273), late-Tumor status group(T3 or T4, $\mathrm{n}=93$ ), low pathological grading group( $\mathrm{G} 1$ or $\mathrm{G} 2, \mathrm{n}=231$ ) and high pathological grading group ( $\mathrm{G} 3$ or $\mathrm{G} 4$, $n=133)$.

\section{Discussion}

The occurrence, development and treatment of liver cancer has become a research hotspot[20-23]. Previously, the treatment and prognostic prediction of patients were established according to tumor size, pathological grading , and lymph node metastasis[24-25]. In reality, however, due to the heterogeneity of 
liver cancer patients, these traditional indicators are often unable to play their roles as predictive indicators[26]. Tumor mutational burden (TMB) is a new biomarker for prediction of response to PD-(L)1 treatment[27-28]. It has also been reported that high TMB predicts poor prognosis in liver cancer patients[29].

LncRNAs has recently been shown to be closely related to the occurrence and development of tumors and abnormal expression of some Incrnas can be prognostic markers in tumor patients[30-35]. With the development of research, people realize that IncrNA also plays an important role in Tumor mutational burden (TMB) [36]. For example, IncRNA DDSR1 participates in DNA damage Response (DDR), and DDR variation will lead to TMB variation[37] . LncRNA NORAD helps to maintain genomic stability[38], which also affects TMB size. How to identify TMB-related Incrnas and their clinical significance for cancer remains to be explored. Therefore, we matched the expression level of IncRNA with the tumor mutation phenotype to identify TMB-related IncRNA. Then we identified 514 TMB-related Incrnas in this computational frame in liver cancer. In addition, on the basis of these TMB-related Incrnas, we built an IncRNA signature (TMBLncSig) to use these IncRNAs to predict the clinical outcome of patients with liver cancer . In the training set, TMBLncSig can well divide patients into low risk group with good prognosis and high risk group with poor prognosis, and there is a significant difference in TMB level between the two groups, which can also be observed in the independent verification set. This suggests that TMBLncSig is not only predictive of prognosis, but also an indicator of TMB levels in patients with liver cancer.

"TP53 is the most frequently mutated gene in human cancer."[39] TP53 mutations occur in more than half of human tumors[40]. In this study TP53 mutation ratio was significantly different among patients in the high-risk group and the low-risk group. The higher score of the patients obtained from TMBLncSig indicated a higher TP53 mutation ratio.This suggests that TMBLncSig can predict TP53 mutation status to a certain extent. Moreover, it was observed that both the TP53 wild-type patients tand TP53 mut-type patients could be distinguished by TMBLncSig with different prognostic outcomes. The prognosis of both TP53 wild-type and TP53 mut-type patients was better in the low-risk group than in the high-risk group.TMBLncSig may be more capable of predicting prognosis than TP53 mutation status alone and it can identify the TP53 wild - type and TP53 mut -type patients in different intermediate subtype. Although our study, such as the construction of TMBLncSig model, can assess the TMB level of patients to a certain extent and provide a feasible way to predict the prognosis of patients, it still has certain limitations and needs further study. TMBLncSig has been verified internally by TCGA data sets, but it still needs to verify its robustness in more independent data sets and different echnology platforms. This study is based on the computational frame of TMB level differences,therefore, the regulation mechanism of TMBLncSig for prognosis and Tumor Mutational burden needs further experimental discovery.

\section{Conclusion}

LncRNAs related to TMB was identified based on TCGA database, which provided resources and methods for further research on the role of IncRNAs in TMB.A TMB-derived IncRNA Signature (TMBLncSig) was 
established by combining the expression levels of these Incrnas, somatic mutation files and clinical information in patients with liver cancer.TMBLncSig can be used as a prognostic marker for liver cancer patients and has been validated in independent test set.In this study, TMBLncSig is of great significance to the TMB level of liver cancer patients and has a certain guiding role in predicting the prognosis of patients.

\section{Declarations}

\section{Declaration of interests}

The authors declare no potential conflict of interest.

\section{Authors' contributions}

Conception and design: Yao Wang

Acquisition of data: Chaisheng Tan

Manuscript writing: Yao Wang

Final approval of manuscript: All authors.

\section{References}

1. Sia D, Villanueva A, Friedman SL, Llovet JM. Liver Cancer Cell of Origin, Molecular Class, and Effects on Patient Prognosis. Gastroenterology. 2017 Mar;152(4):745-761. doi:

10.1053/j.gastro.2016.11.048. Epub 2016 Dec 30. PMID: 28043904.

2. Affo S, Yu LX, Schwabe RF. The Role of Cancer-Associated Fibroblasts and Fibrosis in Liver Cancer. Annu Rev Pathol. 2017 Jan 24;12:153-186. doi: 10.1146/annurev-pathol-052016-100322. Epub 2016 Dec 5. PMID: 27959632; PMCID: PMC5720358.

3. Choucair K, Morand S, Stanbery L, Edelman G, Dworkin L, Nemunaitis J. TMB: a promising immuneresponse biomarker, and potential spearhead in advancing targeted therapy trials. Cancer Gene Ther. 2020 Dec;27(12):841-853. doi: 10.1038/s41417-020-0174-y. Epub 2020 Apr 28. PMID: 32341410.

4. Shrestha R, Prithviraj P, Anaka M, Bridle KR, Crawford DHG, Dhungel B, Steel JC, Jayachandran A. Monitoring Immune Checkpoint Regulators as Predictive Biomarkers in Hepatocellular Carcinoma. Front Oncol. 2018 Jul 13;8:269. doi: 10.3389/fonc.2018.00269. PMID: 30057891; PMCID: PMC6053505.

5. Derrien T, Johnson R, Bussotti G, Tanzer A, Djebali S, Tilgner H, Guernec G, Martin D, Merkel A, Knowles DG, Lagarde J, Veeravalli L, Ruan X, Ruan Y, Lassmann T, Carninci P, Brown JB, Lipovich L, Gonzalez JM, Thomas M, Davis CA, Shiekhattar R, Gingeras TR, Hubbard TJ, Notredame C, Harrow J, Guigó R. The GENCODE v7 catalog of human long noncoding RNAs: analysis of their gene structure, 
evolution, and expression. Genome Res. 2012 Sep;22(9):1775-89. doi: 10.1101/gr.132159.111. PMID: 22955988; PMCID: PMC3431493.

6. Tano K, Akimitsu N. Long non-coding RNAs in cancer progression. Front Genet. 2012 Oct 24;3:219. doi: 10.3389/fgene.2012.00219. PMID: 23109937; PMCID: PMC3479403.

7. Weidle UH, Birzele F, Kollmorgen G, Rüger R. Long Non-coding RNAs and their Role in Metastasis. Cancer Genomics Proteomics. 2017 May-Jun;14(3):143-160. doi: 10.21873/cgp.20027. PMID: 28446530; PMCID: PMC5420816.

8. Huarte M. The emerging role of IncRNAs in cancer. Nat Med. 2015 Nov;21(11):1253-61. doi: 10.1038/nm.3981. PMID: 26540387.

9. Lukas J, Altmeyer M. A IncRNA to repair DNA. EMBO Rep. 2015 Nov;16(11):1413-4. doi: 10.15252/embr.201541309. Epub 2015 Sep 29. PMID: 26420434; PMCID: PMC4641492.

10. Lee S, Kopp F, Chang TC, Sataluri A, Chen B, Sivakumar S, Yu H, Xie Y, Mendell JT. Noncoding RNA NORAD Regulates Genomic Stability by Sequestering PUMILIO Proteins. Cell. 2016 Jan 14;164(12):69-80. doi: 10.1016/j.cell.2015.12.017. Epub 2015 Dec 24. PMID: 26724866; PMCID: PMC4715682.

11. Wang Z, Jensen MA, Zenklusen JC. A Practical Guide to The Cancer Genome Atlas (TCGA). Methods Mol Biol. 2016;1418:111-41. doi: 10.1007/978-1-4939-3578-9_6. PMID: 27008012.

12. Silva TC, Colaprico A, Olsen C, D'Angelo F, Bontempi G, Ceccarelli M, Noushmehr H. TCGA Workflow: Analyze cancer genomics and epigenomics data using Bioconductor packages. F1000Res. $2016 \mathrm{Jun}$ 29;5:1542. doi: 10.12688/f1000research.8923.2. PMID: 28232861; PMCID: PMC5302158.

13. Cunningham F, Amode MR, Barrell D, Beal K, Billis K, Brent S, Carvalho-Silva D, Clapham P, Coates G, Fitzgerald S, Gil L, Girón CG, Gordon L, Hourlier T, Hunt SE, Janacek SH, Johnson N, Juettemann T, Kähäri AK, Keenan S, Martin FJ, Maurel T, McLaren W, Murphy DN, Nag R, Overduin B, Parker A, Patricio M, Perry E, Pignatelli M, Riat HS, Sheppard D, Taylor K, Thormann A, Vullo A, Wilder SP, Zadissa A, Aken BL, Birney E, Harrow J, Kinsella R, Muffato M, Ruffier M, Searle SM, Spudich G, Trevanion SJ, Yates A, Zerbino DR, Flicek P. Ensembl 2015. Nucleic Acids Res. 2015 Jan;43(Database issue):D662-9. doi: 10.1093/nar/gku1010. Epub 2014 Oct 28. PMID: 25352552; PMCID: PMC4383879.

14. Chalmers ZR, Connelly CF, Fabrizio D, Gay L, Ali SM, Ennis R, Schrock A, Campbell B, Shlien A, Chmielecki J, Huang F, He Y, Sun J, Tabori U, Kennedy M, Lieber DS, Roels S, White J, Otto GA, Ross JS, Garraway L, Miller VA, Stephens PJ, Frampton GM. Analysis of 100,000 human cancer genomes reveals the landscape of tumor mutational burden. Genome Med. 2017 Apr 19;9(1):34. doi: 10.1186/s13073-017-0424-2. PMID: 28420421; PMCID: PMC5395719.

15. Kimes PK, Liu Y, Neil Hayes D, Marron JS. Statistical significance for hierarchical clustering. Biometrics. 2017 Sep;73(3):811-821. doi: 10.1111/biom.12647. Epub 2017 Jan 18. PMID: 28099990; PMCID: PMC5708128.

16. Ranstam J, Cook JA. Kaplan-Meier curve. Br J Surg. 2017 Mar;104(4):442. doi: 10.1002/bjs.10238. PMID: 28199017. 
17. Lytsy P. P in the right place: Revisiting the evidential value of P-values. J Evid Based Med. 2018 Nov;11(4):288-291. doi: 10.1111/jebm.12319. Epub 2018 Nov 5. PMID: 30398018; PMCID: PMC6587984.

18. Carter JV, Pan J, Rai SN, Galandiuk S. ROC-ing along: Evaluation and interpretation of receiver operating characteristic curves. Surgery. 2016 Jun;159(6):1638-1645. doi:

10.1016/j.surg.2015.12.029. Epub 2016 Mar 5. PMID: 26962006.

19. Ritchie ME, Phipson B, Wu D, Hu Y, Law CW, Shi W, Smyth GK. limma powers differential expression analyses for RNA-sequencing and microarray studies. Nucleic Acids Res. 2015 Apr 20;43(7):e47. doi: 10.1093/nar/gkv007. Epub 2015 Jan 20. PMID: 25605792; PMCID: PMC4402510.

20. Lu Y, Min Z, Qin A, Wu J, Jiang X, Qiao Z. Role of miR-18a and miR-25 disruption and its mechanistic pattern in progression of liver cancer. 3 Biotech. 2020 Feb;10(2):74. doi: 10.1007/s13205-020-20642. Epub 2020 Jan 28. PMID: 32051807; PMCID: PMC6987266.

21. Lan S, Lin Z, Zhang D, Zeng Y, Liu X. Photocatalysis Enhancement for Programmable Killing of Hepatocellular Carcinoma through Self-Compensation Mechanisms Based on Black Phosphorus Quantum-Dot-Hybridized Nanocatalysts. ACS Appl Mater Interfaces. 2019 Mar 13;11(10):9804-9813. doi: 10.1021/acsami.8b21820. Epub 2019 Feb 27. PMID: 30773883.

22. Dubbelboer IR, Sjögren E, Lennernäs H. Porcine and Human In Vivo Simulations for DoxorubicinContaining Formulations Used in Locoregional Hepatocellular Carcinoma Treatment. AAPS J. 2018 Aug 30;20(6):96. doi: 10.1208/s12248-018-0251-4. PMID: 30167825.

23. Pei $Y$, Zhang T, Renault $V$, Zhang $X$. An overview of hepatocellular carcinoma study by omics-based methods. Acta Biochim Biophys Sin (Shanghai). 2009 Jan;41(1):1-15. doi: 10.1093/abbs/gmn001. PMID: 19129945.

24. Zhang W, Wang X, Jiang R, Hou J, Mu X, Li G, Sun B. Effect of Tumor Size on Cancer-Specific Survival in Small Hepatocellular Carcinoma. Mayo Clin Proc. 2015 Sep;90(9):1187-95. doi: 10.1016/j.mayocp.2015.06.018. Epub 2015 Jul 29. PMID: 26231292.

25. Llovet JM, Brú C, Bruix J. Prognosis of hepatocellular carcinoma: the BCLC staging classification. Semin Liver Dis. 1999;19(3):329-38. doi: 10.1055/s-2007-1007122. PMID: 10518312.

26. Lin DC, Mayakonda A, Dinh HQ, Huang P, Lin L, Liu X, Ding LW, Wang J, Berman BP, Song EW, Yin D, Koeffler HP. Genomic and Epigenomic Heterogeneity of Hepatocellular Carcinoma. Cancer Res. 2017 May 1;77(9):2255-2265. doi: 10.1158/0008-5472.CAN-16-2822. Epub 2017 Feb 20. PMID: 28302680; PMCID: PMC5413372.

27. Zhu J, Zhang T, Li J, Lin J, Liang W, Huang W, Wan N, Jiang J. Association Between Tumor Mutation Burden (TMB) and Outcomes of Cancer Patients Treated With PD-1/PD-L1 Inhibitions: A MetaAnalysis. Front Pharmacol. 2019 Jun 14;10:673. doi: 10.3389/fphar.2019.00673. PMID: 31258479; PMCID: PMC6587434.

28. Goodman AM, Kato S, Bazhenova L, Patel SP, Frampton GM, Miller V, Stephens PJ, Daniels GA, Kurzrock R. Tumor Mutational Burden as an Independent Predictor of Response to Immunotherapy in 
Diverse Cancers. Mol Cancer Ther. 2017 Nov;16(11):2598-2608. doi: 10.1158/1535-7163.MCT-170386. Epub 2017 Aug 23. PMID: 28835386; PMCID: PMC5670009.

29. Romero D. TMB is linked with prognosis. Nat Rev Clin Oncol. 2019 Jun;16(6):336. doi: 10.1038/s41571-019-0206-4. PMID: 30932077.

30. Bhan A, Soleimani M, Mandal SS. Long Noncoding RNA and Cancer: A New Paradigm. Cancer Res. 2017 Aug 1;77(15):3965-3981. doi: 10.1158/0008-5472.CAN-16-2634. Epub 2017 Jul 12. PMID: 28701486.

31. Li J, Meng H, Bai Y, Wang K. Regulation of IncRNA and Its Role in Cancer Metastasis. Oncol Res. 2016;23(5):205-17. doi: 10.3727/096504016X14549667334007. PMID: 27098144.

32. Schmitt AM, Chang HY. Long Noncoding RNAs in Cancer Pathways. Cancer Cell. 2016 Apr 11;29(4):452-463. doi: 10.1016/j.ccell.2016.03.010. PMID: 27070700; PMCID: PMC4831138.

33. Ma Y, Zhang J, Wen L, Lin A. Membrane-lipid associated IncRNA: A new regulator in cancer signaling. Cancer Lett. 2018 Apr 10;419:27-29. doi: 10.1016/j.canlet.2018.01.008. Epub 2018 Jan 9. PMID: 29330108.

34. Abbastabar M, Sarfi M, Golestani A, Khalili E. IncRNA involvement in hepatocellular carcinoma metastasis and prognosis. EXCLI J. 2018 Sep 4;17:900-913. doi: 10.17179/excli2018-1541. PMID: 30564069; PMCID: PMC6295623.

35. Klingenberg M, Matsuda A, Diederichs S, Patel T. Non-coding RNA in hepatocellular carcinoma: Mechanisms, biomarkers and therapeutic targets. J Hepatol. 2017 Sep;67(3):603-618. doi: 10.1016/j.jhep.2017.04.009. Epub 2017 Apr 22. PMID: 28438689.

36. Liu Z, Mi M, Li X, Zheng X, Wu G, Zhang L. A IncRNA prognostic signature associated with immune infiltration and tumour mutation burden in breast cancer. J Cell Mol Med. 2020 Nov;24(21):1244412456. doi: 10.1111/jcmm.15762. Epub 2020 Sep 23. PMID: 32967061; PMCID: PMC7687003.

37. Lukas J, Altmeyer M. A IncRNA to repair DNA. EMBO Rep. 2015 Nov;16(11):1413-4. doi: 10.15252/embr.201541309. Epub 2015 Sep 29. PMID: 26420434; PMCID: PMC4641492.

38. Munschauer M, Nguyen CT, Sirokman K, Hartigan CR, Hogstrom L, Engreitz JM, Ulirsch JC, Fulco CP, Subramanian V, Chen J, Schenone M, Guttman M, Carr SA, Lander ES. The NORAD IncRNA assembles a topoisomerase complex critical for genome stability. Nature. 2018 Sep;561(7721):132136. doi: 10.1038/s41586-018-0453-z. Epub 2018 Aug 27. Erratum in: Nature. 2018 Oct 2;: PMID: 30150775.

39. Baker SJ, Fearon ER, Nigro JM, Hamilton SR, Preisinger AC, Jessup JM, vanTuinen P, Ledbetter DH, Barker DF, Nakamura Y, White R, Vogelstein B. Chromosome 17 deletions and p53 gene mutations in colorectal carcinomas. Science. 1989 Apr 14;244(4901):217-21. doi: 10.1126/science.2649981. PMID: 2649981.

40. Leroy B, Anderson M, Soussi T. TP53 mutations in human cancer: database reassessment and prospects for the next decade. Hum Mutat. 2014 Jun;35(6):672-88. doi: 10.1002/humu.22552. PMID: 24665023. 


\section{Tables}

Table 1: The clinical and pathological characteristics of the patients in TCGA data sets

\begin{tabular}{|c|c|c|c|c|c|}
\hline Covariates & Type & TCGA set & Test set & Train set & Pvalue \\
\hline \multirow[t]{2}{*}{ Age, no (\%) } & $₫ 60$ & $192(52.03 \%)$ & $92(50 \%)$ & $100(54.05 \%)$ & $0.4996^{\mathrm{a}}$ \\
\hline & $\leq 60$ & $177(47.97 \%)$ & $92(50 \%)$ & $85(45.95 \%)$ & \\
\hline \multirow[t]{2}{*}{ Sex, no (\%) } & FEMALE & $121(32.79 \%)$ & $64(34.78 \%)$ & $57(30.81 \%)$ & $0.4829^{a}$ \\
\hline & MALE & $248(67.21 \%)$ & $120(65.22 \%)$ & 128(69.19\%) & \\
\hline \multirow[t]{3}{*}{ T, no (\%) } & $\mathrm{T} 1-\mathrm{T} 2$ & $273(73.98 \%)$ & $131(71.2 \%)$ & 142(76.76\%) & $0.307^{a}$ \\
\hline & T3-T4 & $93(25.2 \%)$ & $51(27.72 \%)$ & $42(22.7 \%)$ & \\
\hline & unknow & $3(0.81 \%)$ & $2(1.09 \%)$ & $1(0.54 \%)$ & \\
\hline \multirow[t]{3}{*}{ N, no (\%) } & NO & $252(68.29 \%)$ & $125(67.93 \%)$ & $127(68.65 \%)$ & $0.6143^{a}$ \\
\hline & $\mathrm{N} 1$ & $4(1.08 \%)$ & $3(1.63 \%)$ & $1(0.54 \%)$ & \\
\hline & unknow & $113(30.62 \%)$ & $56(30.43 \%)$ & $57(30.81 \%)$ & \\
\hline \multirow[t]{3}{*}{$\mathrm{M}, \mathrm{no}(\%)$} & MO & $265(71.82 \%)$ & 133(72.28\%) & 132(71.35\%) & $0.9579^{a}$ \\
\hline & M1 & $4(1.08 \%)$ & $2(1.09 \%)$ & $2(1.08 \%)$ & \\
\hline & unknow & $100(27.1 \%)$ & $49(26.63 \%)$ & $51(27.57 \%)$ & \\
\hline \multirow[t]{3}{*}{ Stage, no (\%) } & Stage I-II & $255(69.11 \%)$ & $123(66.85 \%)$ & 132(71.35\%) & $0.2103^{\mathrm{a}}$ \\
\hline & Stage III-IV & $90(24.39 \%)$ & $51(27.72 \%)$ & $39(21.08 \%)$ & \\
\hline & unknow & $24(6.5 \%)$ & $10(5.43 \%)$ & $14(7.57 \%)$ & \\
\hline \multirow[t]{3}{*}{ Grade, no (\%) } & $\mathrm{G} 1-\mathrm{G} 2$ & $231(62.6 \%)$ & $115(62.5 \%)$ & $116(62.7 \%)$ & $0.8901^{\mathrm{a}}$ \\
\hline & G3-G4 & 133(36.04\%) & $68(36.96 \%)$ & $65(35.14 \%)$ & \\
\hline & unknow & $5(1.36 \%)$ & $1(0.54 \%)$ & $4(2.16 \%)$ & \\
\hline
\end{tabular}

${ }^{\mathrm{a} C h i ~ s q u a r e ~ t e s t ~}$

\section{Figures}

Figure 1 
(A) Unsupervised hierarchical clustering analysis was carried out for a total of 374 liver cancer patients using the 514 TMB-related IncRNAs. The green cluster is highTMB-like group, and the red cluster is lowTMB-like group. (B) The TMB levels of the HightMB-like Group and lowtMB-like Group are shown using a boxplot. The TMB level of hightMB-like Group is significantly higher than that of lowtMB-like Group.

\section{Figure 2}

(A) The prognostic value of TMBLncSig score in training set (Kaplan-Meier Plotter). Statistical analysis was performed using the log-rank test and univariate Cox analysis. (B) Time-dependent ROC curves analysis of the TMBLncSig at 1,2,3,4 and 5 years. (C) With The increase of TMBLncSig Score, The distribution of The TMB level also presents an upward trend . (D) In the boxplot, red represents the highrisk group, blue represents the low-risk group, and the TMB level of the high-risk group is significantly higher than that of the low-risk group.Statistical analysis was performed using the Mann-Whitney $U$ test.

\section{Figure 3}

Performance evaluation of the TMBLncSig in the TCGA set. Kaplan Meier estimates of OS of liver cancer patients with low or high risk predicted by the TMBLncSig in the testing set (A) and TCGA set (B). Statistical analysis was performed using the log-rank test and univariate Cox analysis.Time-dependent ROC curves analysis of the TMBLncSig at 1year in train set (C) and TCGA set (D). With The increase of TMBLncSig Score, The distribution of The TMB level also presents an upward trend in train set (E) and TCGA set (F) .In the boxplot, the TMB level of the High-Risk (red) Group is higher than that of the low-risk Group (blue) in the testing set $(G)$ and TCGA set $(H)$. Horizontal lines: median values. Statistical analysis was performed using the Mann Whitney $U$ test.

\section{Figure 4}

(A)The proportion of TP53 mutation in high-risk group and low-risk group in the training set, testing set and the TCGA set.(B)The Kaplan-Meier plot of four groups (TP53 Mutation/high risk group(n=66),TP53 Mutation/low risk group( $n=43)$, TP53 Wild/high risk group( $n=98)$ and TP53 Wild/low risk group( $n=150)$. Statistical analysis was performed using the log-rank test.

\section{Figure 5}

Kaplan-Meier curve analysis of overall survival in high-risk group and low-risk group for young patients (A) and old patients (B). Kaplan-Meier curve analysis of overall survival in high-risk group and low-risk group for early-stage patients (C) and late-stage patients (D).Kaplan-Meier curve analysis of overall 
survival in high-risk group and low-risk group for low pathological grading group(E) and high pathological grading group (F) .Kaplan-Meier curve analysis of overall survival in high-risk group and low-risk group for early-Tumor status group $(G)$, late-Tumor status group $(H)$. Statistical analysis was performed using the log-rank test and univariate Cox analysis

\section{Supplementary Files}

This is a list of supplementary files associated with this preprint. Click to download.

- SupplementaryTables.docx 